\title{
Potential problems arising during international disaster victim identification (DVI) exercises
}

\author{
Roger W. Byard · Calle Winskog
}

Published online: 19 January 2010

(C) Springer Science+Business Media, LLC 2010

Disaster victim identification (DVI) refers to the formal and organised process of identifying multiple bodies after a mass fatality. The number of dead required before DVI processes are initiated varies among jurisdictions, for example it may occur with only four bodies after a vehicle crash, or with many thousands after a tsunami. International DVI procedures are coordinated through Interpol with local authorities generally having control over the site(s) of the disaster, but sometimes with assistance from outside organizations. Guidelines and internationally accepted forms are provided by Interpol for collating both antemortem and post-mortem data. DVI exercises may involve recent natural disasters such as earthquakes, terrorist actions such as suicide bombings, human trafficking accidents, or events that have happened some time ago, as in exhumations of mass graves from previous armed conflict [1].

A variety of problems can occur when large operations are commenced on foreign soil. Given the high level of interest in such exercises amongst forensic professionals there is often quite a strong push for participation when a high profile disaster occurs. This results in rapid mobilization of teams and individuals, sometimes without adequate, or any, consultation with local authorities. This international "DVI industry" may result in deployment of individuals and teams that may include some who are not engaged in current professional practice, and also conversely in significant

R. W. Byard $(\bowtie) \cdot$ C. Winskog

Discipline of Pathology, The University of Adelaide, Level 3

Medical School North Building, Frome Road,

Adelaide 5005, Australia

e-mail: roger.byard@sa.gov.au

R. W. Byard - C. Winskog

Forensic Science SA, 21 Divett Place, Adelaide,

SA 5000, Australia deskilling of those left behind - an undesirable effect on both counts. It is important to ensure that all forensic professionals are trained in this area as it is preferable, where the scale allows, that local disasters are handled by locally-based professionals, as they are the ones who have the best understanding of many of the local issues.

Operating in an external jurisdiction also brings with it complex legal and cultural issues. The impression sometimes given to local authorities is that they are merely being tolerated by the true 'experts' in the field, however the reality is often far from this. Cultural sensitivities are extremely important and every effort should be made for an experienced team leader and team members to be adequately briefed on local religious beliefs, cultural attitudes and practices, and political systems [2]. This may only require the application of common sense, such as consulting regularly with local authorities before any changes in work practices occur, or simply not placing a visitor's flag over a national flag at a work site.

A multidisciplinary approach is essential to the success of these operations with input from representatives of all major professional groups. It is important to maintain this multidisciplinary interaction from the initial planning stages right through to signing off cases at Reconciliation Panel meetings between visiting experts and local authorities. For example, failure to consult with pathologists in the early stages of planning may result in inadequate sampling being undertaken, which then necessitates reprocessing of cases for a second or even a third time, all of which is exceedingly time consuming and expensive. One of the most important philosophies is to 'hasten slowly', as the consequences of errors in identification due to rushed work could outlast the operation by many years, if not decades.

Once in the field it is important that the health of the team is cared for, and this includes both psychological and 
medical support. The importance of this cannot be underestimated and it is the responsibility of an experienced team leader to care for his team and their wellbeing. Having a medical officer attached to each national team should be a requirement, as teams may be working in hot tropical environments without adequate attention being paid to the basics of maintaining adequate hydration, or in dealing with the ever-present risk of infection. Facilities may be extremely rudimentary due to local economic constraints, geographic isolation, or the effects of a natural disaster such as a tsunami destroying infrastructure. Debriefing sessions after such deployments are often quite limited in their scope, duration and number.

A constant problem that is faced when there are a number of teams from multiple countries working in an operation is ensuring that protocols are strictly adhered to and that practices meet professional and ethical standards. It is a requirement that Interpol forms are filled out in English and that the originals are kept at the reconciliation center. However this does not always occur, with languages other then English being used, and only partial and/or copied forms sometimes being submitted. An adequate preparation period, including agreement on practices used for the specific exercise, could prevent and minimize these well-known and often repeated errors. It is also recommended that a clearly understood and simple numbering/ labelling system for bodies and parts is established very early in the process. Relabelling, or the use of multiple numbers for the same body, is a recipe for confusion and error with the distinct possibility of misidentifying bodies and releasing wrong bodies to grieving families. In cases involving foreign nationals the logistics of returning a misidentified body are significant.

An additional problem involves the taking of samples. All samples should be taken in an agreed upon and uniform manner, and submitted through official channels for identification. Teams should not be permitted to sample only individuals from their own countries and send material to their own institutions for identification, as this bypasses protocols and can create significant administrative problems. Once jewellery or personal possessions have been located they should be photographed, described and placed in signed and sealed plastic bags and kept with the body. The practice of placing unmarked and unlabelled jewellery under bodies in their body bags is another example of an incorrect and idiosyncratic practice that should be strongly discouraged. It must also be remembered that the forensic value of personal possessions can be challenged and questioned once they have been taken from a body without proper documentation.

The quality of autopsy examinations being performed is also sometimes a major issue. An example of this has been the practice of some teams of routinely removing fingers and jaws from bodies, ostensibly for identification purposes. Not only is this extremely mutilating but it removes essential features from bodies that may not be able to be identified by other means. There is a danger also that dissected jaws and fingers may be lost or misplaced. It should be the team leader's responsibility to discuss this deviation from normal practices with local authorities to determine an appropriate action.

Despite the quite serious problems that have been encountered in international DVI operations over the years, it must be acknowledged that the process has generally been successful. To ensure that this success continues it will be important for individual countries to maintain their DVI skills on a national level, with regular international consultation. Protocols and training need to be kept up-todate so that the best-trained and equipped teams are available for rapid deployment if required. Clear authority needs to be established at an operation so that individuals and/or teams using idiosyncratic or inappropriate methodologies can be counselled, advised, retrained or, if necessary, dismissed. Above all there must be early and close consultation with local authorities to ensure that operations proceed in full accord with local legal and cultural practices. While encouraging local responsibility in major mass disasters, the global community does have an obligation and responsibility to assist in the process of identification, and this should be undertaken without consideration of any economic or political advantages.

\section{References}

1. Rutty GN, Byard RW, Tsokos M. The tsunami. An environmental mass disaster. Forensic Sci Med Pathol. 2005;1:3-8.

2. Byard RW, Cooke C, Leditschke J. Practical issues involved in setting up temporary mortuaries after mass disasters. Forensic Sci Med Pathol. 2006;2:59-61. 